\title{
Effect Algebras, Presheaves, Non-locality and Contextuality
}

\author{
Sam Staton and Sander Uijlen \\ University of Oxford and Radboud University, Nijmegen
}

\begin{abstract}
Non-locality and contextuality are among the most counterintuitive aspects of quantum theory. They are difficult to study using classical logic and probability theory. In this paper we start with an effect algebraic approach to the study of non-locality and contextuality. We will see how different slices over the category of set valued functors on the natural numbers induce different settings in which non-locality and contextuality can be studied. This includes the Bell, Hardy and Kochen-Specker-type paradoxes. We link this to earlier sheaf theoretic approaches by defining a fully faithful embedding of the category of effect algebras in this presheaf category over the natural numbers.
\end{abstract}

\section{Introduction}

This paper is about generalized theories of probability that allow us to analyze the non-locality and contextuality paradoxes from quantum theory. Informally, the paradoxes have to do with the idea that it might not be possible to explain the outcomes of measurements in a classical way. We proceed by using now-standard techniques for local reasoning in computer science. Partial monoids play a crucial role in 'separation logic' which is a basic framework of locality especially relevant to memory locality (e.g. $[3,4])$. Presheaves on natural numbers have already been used to study local memory (e.g. [16]) and also to study contexts in abstract syntax (e.g. [7]).

The paper is in two parts. In the first we establish new relationships between two generalized theories of probability. In the second we analyze the paradoxes of contextuality using our theories of probability, and we use this to recover earlier formulations of them in different frameworks.

\subsection{Generalized probability measures}

Recall that a finite measurable space $(X, \Omega)$ comprises a finite set $X$ and a sub-Boolean algebra $\Omega$ of the powerset $\Omega \subseteq \mathcal{P}(X)$, and recall:

Definition 1 A probability distribution on a finite measurable space $(X, \Omega)$ is a function $p: \Omega \rightarrow[0,1]$ such that $p(X)=1$ and if $A_{1} \ldots A_{n}$ are disjoint sets in $\Omega$, then $\sum_{i=1}^{n} p\left(A_{i}\right)=p\left(\bigcup_{i=1}^{n} A_{i}\right)$.

We now analyze this definition to propose two general notions of probability measure. (NB. We will focus on finite probability spaces, because this is sufficient for our examples. We intend to return to infinite spaces in future work.) 
Partial monoids. Our first generalization involves partial monoids. Notice that the conditions on the probability distribution $p: \Omega \rightarrow[0,1]$ do not involve the space $\mathcal{P}(X)$. We only used the disjoint union structure of $\Omega$. More generally, we can define a pointed partial commutative monoid (PPCM) to be a structure $(E, \oslash, 0,1)$ where $\oslash: E \times E \rightarrow E$ is a commutative, associative partial binary operation with a unit 0 . Then $(\Omega, \uplus, \emptyset, X)$ and the interval $([0,1],+, 0,1)$ are PPCMs. A probability distribution is now the same thing as a PPCM homomorphism, $(\Omega, \uplus, \emptyset, X) \rightarrow([0,1],+, 0,1)$. Thus PPCMs are a candidate for a generalized probability theory. (This is a long-established position; see e.g. [6].)

Functors. Our second generalization goes as follows. Every finite Boolean algebra $\Omega$ is isomorphic to one of the form $\mathcal{P}(N)$ for a finite set $N$, called the atoms of $\Omega$. Now, a probability distribution $p: \Omega \rightarrow[0,1]$ is equivalently given by a function $q: N \rightarrow[0,1]$ such that $\sum_{a \in N} q(a)=1$. Let

$$
D(N)=\left\{q: N \rightarrow[0,1] \mid \sum_{a \in N} q(a)=1\right\}
$$

be the set of all distributions on a finite set $N$. It is well-known that $D$ extends to a functor $D:$ FinSet $\rightarrow$ Set. The Yoneda lemma gives a bijection between distributions in $D(N)$ and natural transformations $\operatorname{FinSet}(N,-) \rightarrow D$. Thus we are led to say that a generalized finite measurable space is a functor $F:$ FinSet $\rightarrow$ Set (aka presheaf), and a probability distribution on $F$ is a natural transformation $F \rightarrow D$. (This appears to be a new position.)

Relationship. Our main contribution in Section 2 and 3 is an adjunction between the two kinds of generalized measurable spaces: PPCMs, and presheaves FinSet $\rightarrow$ Set. 'Effect algebras' are a special class of PPCMs [5, 9]. We show that our adjunction restricts to a reflection from effect algebras into presheaves FinSet $\rightarrow$ Set, which gives us a slogan that 'effect algebras are well-behaved generalized finite measurable spaces'.

\subsection{Relating non-locality and contextuality arguments}

In the second part of the paper we investigate three paradoxes from quantum theory, attributed to Bell, Hardy and Kochen-Specker. We justify our use of effect algebras and presheaves by establishing relationships with earlier work by Abramsky and Brandenburger [1] and Hamilton, Isham and Butterfield [10]. For the purposes of introduction, we focus on the Bell paradox, and we focus on the mathematics. (Some physical intuitions are given in Section 4.)

The Bell paradox in terms of effect algebras and presheaves. As we show, the Bell scenario can be understood as a morphism of effect algebras $E \stackrel{t}{\rightarrow}[0,1]$, i.e., a generalized probability distribution. The paradox is that although this has a quantum realization, in that it factors through $\operatorname{Proj}(\mathcal{H})$, the projections on a 
Hilbert space $\mathcal{H}$, it has no explanation in classical probability theory, in that there it does not factor through a given Boolean algebra $\Omega$. Informally:

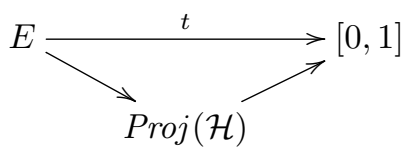

but

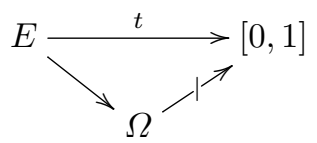

Relationship with earlier sheaf-theoretic work on the Bell paradox. In [1], Abramsky and Brandenburger have studied Bell-type scenarios in terms of presheaves. We recover their results from our analysis in terms of generalized probability theory. Our first step is to notice that effect algebras essentially fully embed in the functor category [FinSet $\rightarrow$ Set]. We step even closer by recalling the slice category construction. This is a standard technique of categorical logic for working relative to a particular object. As we explain in Section 4, the slice category $[$ FinSet $\rightarrow$ Set $] / \Omega$ is again a presheaf category. It is more-or-less the category used in [1]. Moreover, our non-factorization (2) transports to the slice category: $\Omega$ becomes terminal, and $E$ is a subterminal object. Thus the non-factorization in diagram (2) can be phrased in the sheaf-theoretic language of Abramsky and Brandenburger: 'the family $t$ has no global section'.

Other paradoxes Alongside the Bell paradox we study two other paradoxes:

- The Hardy paradox is similar to the Bell paradox, except that it uses possibility rather than probability. We analyze this by replacing the unit interval $([0,1],+, 0,1)$ by the PPCM $(\{0,1\}, \vee, 0,1)$ where $\vee$ is bitwise-or. Although this monoid is not an effect algebra, everything still works and we are able to recover the analysis of the Hardy paradox by Abramsky and Brandenburger.

- The Kochen-Specker paradox can be understood as saying that there is no PPCM morphism

$$
\operatorname{Proj}(\mathcal{H}) \rightarrow(\{0,1\}, \otimes, 0,1)
$$

with $\operatorname{dim} . \mathcal{H} \geq 3$ and where $\oslash$ is like bitwise-or, except that $1 \otimes 1$ is undefined. Now, the slice category $[$ FinSet $\rightarrow \operatorname{Set}] / \operatorname{Proj}(\mathcal{H})$ is again a presheaf category, and it is more-or-less the presheaf category used by Hamilton, Isham and Butterfield. The non-existence of a homomorphism (3) transports to this slice category: $\operatorname{Proj}(\mathcal{H})$ becomes the terminal object, and $(\{0,1\}, \oslash, 0,1)$ becomes the so-called 'spectral presheaf'. We are thus able to rephrase the non-existence of a homomorphism (3) in the same way as Hamilton, Isham and Butterfield [10]: 'the spectral presheaf does not have a global section'.

Summary Motivated by techniques for locality in computer science, we have developed a framework for generalized probability theory based on effect algebras and presheaves. The relevance of the framework is demonstrated by the paradoxes of non-locality and contextuality, which arise as diagrams in one fundamental adjunction. Different analyses in the literature use different presheaf categories, but these all arise from our analysis by taking slice categories. 


\section{Pointed Partial Commutative Monoids}

Definition $2 A$ pointed partial commutative monoid (PPCM) $(E, 0,1, \emptyset)$ consists of a set $E$ with a special element $0 \in E$, a chosen point $1 \in E$ and a partial function $\emptyset: E \times E \rightarrow E$, such that for all $x, y, z \in E$ we have:

1. If $x \otimes y$ is defined, then $y \emptyset x$ is also defined and $x \otimes y=y \otimes x$.

2. $x \otimes 0$ is always defined and $x=x \otimes 0$.

3. If $x \otimes y$ and $(x \otimes y) \emptyset z$ are defined, then $y \oslash z$ and $x \otimes(y \otimes z)$ are defined and $(x \otimes y) \oslash z=x \otimes(y \oplus z)$.

We write $x \perp y$ (say $x$ is perpendicular to $y$ ), if $x \otimes y$ is defined. When we write $x \otimes y$, we tacitly assume $x \perp y$. We refer to $x \otimes y$ as the sum of $x$ and $y$.

A morphism $f: E \rightarrow F$ of PPCMs is a map such that $f(0)=0, f(1)=1$ and $f(a \oslash b)=f(a) \oslash f(b)$ whenever $a \perp b$. This entails the category PPCM.

Definition 3 An effect algebra $(E, 0, \oslash, 1)$ is a $\operatorname{PPCM}(E, 0, \otimes, 1)$ such that

1. For every $x \in E$ there exists a unique $x^{\perp}$ such that $x \perp x^{\perp}$ and $x \otimes x^{\perp}=1$.

2. $x \perp 1$ implies $x=0$.

We call $x^{\perp}$ the 'orthocomplement of $x$ '. PPCM morphisms between effect algebras always preserve orthocomplements. We denote by EA the full subcategory of PPCM whose objects are effect algebras.

Example $4-$ We will consider the set $2=\{0,1\}$ as a PPCM in two ways.

- The initial PPCM $(2, \emptyset, 0,1)$ has $0 \emptyset 0=0$ and $1 \otimes 0=0 \otimes 1=1$; this is an effect algebra.

- The monoid $(2, \vee, 0,1)$ with $0 \vee 0=0$ and $1 \vee 0=0 \vee 1=1 \vee 1=1$; this is not an effect algebra.

- Any Boolean algebra $(B, \vee, \wedge, 0,1)$ is an effect algebra $(B, \oslash, 0,1)$ where $x \perp$ $y$ iff $x \wedge y=0$, and then $x \otimes y \stackrel{\text { def }}{=} x \vee y$. A function between Boolean algebras is a Boolean algebra homomorphism iff it is a PPCM morphism.

- The projections on a Hilbert space form an effect algebra $(\operatorname{Proj}(\mathcal{H}),+, 0,1)$ where $p \perp q$ if their ranges are orthogonal.

- The unit interval $([0,1],+, 0,1)$ is an effect algebra when $x \perp y$ iff $x+y \leq 1$.

\section{Presheaves and tests}

In this section we consider a different notion of generalized probability space. Recall that for any finite set $N$ we have a set $D(N)$ of distributions (Equation (1)). This construction is functorial in $N$. Consider the category $\mathbb{N}$, the skeleton of FinSet, whose objects are natural numbers considered as sets, $N=\{1, \ldots, n\}$, and whose morphisms are functions. Then $D: \mathbb{N} \rightarrow$ Set, with $((D f)(q))(i)=$ $\sum_{j \in f^{-1}(i)} q(j)$. 
This leads us to a notion of generalized probability space via the Yoneda lemma. Write $\operatorname{Set}^{\mathbb{N}}$ for the category of functors $\mathbb{N} \rightarrow \operatorname{Set}($ aka 'covariant presheaves') and natural transformations. The Yoneda lemma says $D(N) \cong \operatorname{Set}^{\mathbb{N}}(\mathbb{N}(N,-), D)$. More generally we can thus understand natural transformations $F \rightarrow D$ as 'distributions' on a functor $F \in \mathbf{S e t}^{\mathbb{N}}$.

To make a connection between presheaves and PPCMs and effect algebras we recall the notion of test.

Definition 5 Let $E$ be a PPCM. An $n$-test in $E$ is an $n$-tuple $\left(e_{1}, \ldots, e_{n}\right)$ of elements in $E$ such that $e_{1} \otimes \ldots \otimes e_{n}=1$.

The tests of a PPCM $E$ form a presheaf $T(E) \in \operatorname{Set}^{\mathbb{N}}$, where $T(E)(N)$ is the set of $n$-tests in $E$, and if $f: N \rightarrow M$ is a function then

$$
T(E)(f)\left(e_{1}, \ldots, e_{n}\right)=\left(\bigotimes_{i \in f^{-1}(j)} e_{i}\right)_{j=1, \ldots, m}
$$

This extends to a functor $T: \mathbf{P P C M} \rightarrow \mathbf{S e t}^{\mathbb{N}}$. If $\psi: E \rightarrow A$ is a PPCM morphism, then we obtain the natural transformation $T(\psi)$ with components $T(\psi)_{N}\left(e_{1}, \ldots, e_{n}\right)=\left(\psi\left(e_{1}\right), \ldots, \psi\left(e_{n}\right)\right)$. (See also [12, Def. 6.3].)

Example $6-T(2, \otimes, 0,1) \in \mathbf{S e t}^{\mathbb{N}}$ is the inclusion: $(T(2, \otimes, 0,1))(N)=N$.

$-T(2, \vee, 0,1) \in \mathbf{S e t}^{\mathbb{N}}$ is the non-empty powerset functor: $(T(2, \vee, 0,1))(N)=$ $\{S \subseteq N \mid S \neq \emptyset\}$.

- Any finite Boolean algebra $(B, \vee, \wedge, 0,1)$ is of the form $\mathcal{P}(N)$ for a finite set $N$; we have $T(B, \otimes, 0,1)=\mathbb{N}(N,-)$, the representable functor.

- For the unit interval, $T([0,1],+, 0,1)=D$, the distribution functor.

Our main result in this section is that the test functor essentially exhibits effect algebras as a full subcategory of $\mathbf{S e t}^{\mathbb{N}}$.

Theorem 7 The induced function $T_{A, B}: \operatorname{PPCM}(A, B) \rightarrow \operatorname{Set}^{\mathbb{N}}(T A, T B)$ is a bijection when $A$ is an effect algebra.

Proof (summary). Since $A$ is an effect algebra, every element $a \in A$ is part of a 2 -test $\left(a, a^{\perp}\right)$. It is then clear that $T_{A, B}$ is injective. Now suppose we have some natural transformation $\mu: T(A) \rightarrow T(B)$. The map $\psi_{\mu}: A \rightarrow B$ defined by $\psi_{\mu}(a)=x$, where $\left(x, x^{\perp}\right)=\mu_{2}\left(a, a^{\perp}\right)$ has the property that $T\left(\psi_{\mu}\right)=\mu$.

Corollary 8 The restriction to effect algebras, $T: \mathbf{E A} \rightarrow$ Set $^{\mathbb{N}}$, is full and faithful.

We remark that a more abstract way to view the test functor is through the framework of nerves and realizations. For any natural number $N$ the powerset $\mathcal{P}(N)$ is a Boolean algebra and hence an effect algebra. This extends to a functor $\mathcal{P}: \mathbb{N}^{o p} \rightarrow$ PPCM . The test functor $T$ has a left adjoint, which is the left Kan extension of $\mathcal{P}$ along the Yoneda embedding. (This follows from Theorem 2 of [15, Ch. I.5]; PPCM is cocomplete by [2, Theorem 3.36].) Theorem 7 can be phrased 'the counit is an isomorphism at effect algebras', and Corollary 8 can be phrased 'finite Boolean algebras are dense in effect algebras'. 


\section{Non-locality and contextuality}

In probability theory, questions of contextuality arise from the problem that the joint probability distribution for all outcomes of all measurements may not exist. We suppose a simple framework where Alice and Bob each have a measurement device with two settings. For simplicity we suppose that the device will emit 0 or 1 , as the outcome of a measurement. We write $\mathrm{a}_{0}: 0$ for 'Alice measured 0 with setting $\mathrm{a}_{0}$ ', $\mathrm{b}_{1}: 0$ for 'Bob measured 0 with setting $\mathrm{b}_{1}$ ', and so on. To model this in classical probability theory we would consider a sample space $S_{\mathrm{A}}$ for Alice whose elements are functions $\left\{\mathrm{a}_{0}, \mathrm{a}_{1}\right\} \rightarrow\{0,1\}$, i.e., assignments of outcomes to measurements. Similarly we have a sample space $S_{\mathrm{B}}$ for Bob. We would then consider a joint probability distribution on $S_{\mathrm{A}}$ and $S_{\mathrm{B}}$.

In this model, we implicitly assume that Alice and Bob can not signal to each other. That is to say, for any joint distribution we can define marginal distributions each for Alice and Bob. However, the classical model does include an assumption: that Alice is able to record the outcome of the measurement in both settings. In reality, and in quantum physics, once Alice has recorded an outcome using one measurement setting, she cannot then know what the outcome would have been using the other measurement setting. Effect algebras provide a way to describe a kind of probability distribution that takes this measure-onlyonce phenomenon into account.

The non-locality 'paradox' is as follows: there are probability distributions in this effect algebraic sense (without signalling), which are physically realizable, but cannot be explained in a classical probability theory without signalling.

The main purpose of this section is not to study non-locality and contextuality in different systems, but rather to give a general framework to study them. We use this to recover earlier frameworks.

\subsection{Bimorphisms, joint distributions, and tables}

It is convenient to first introduce a notion of bimorphism, which captures the notion of a probability distribution on joint measurements. Later we will see that bimorphisms are classified by a tensor product.

Definition 9 Let $A, B$ and $C$ be pointed partial commutative monoids. $A$ bimorphism $A, B \rightarrow C$ is a function $f: A \times B \rightarrow C$ such that for all a, $a_{1}, a_{2} \in A$ and $b, b_{1}, b_{2} \in B$ with $a_{1} \perp a_{2}$ and $b_{1} \perp b_{2}$ we have

$$
\begin{array}{cl}
f\left(a, b_{1} \oslash b_{2}\right)=f\left(a, b_{1}\right) \otimes f\left(a, b_{2}\right) & f\left(a_{1} \oslash a_{2}, b\right)=f\left(a_{1}, b\right) \oslash f\left(a_{2}, b\right) \\
f(a, 0)=f(0, b)=0 & f(1,1)=1
\end{array}
$$

We now describe the scenario in the introduction to this section using bimorphisms. Let $E_{\mathrm{A}}$ be the effect algebra $\left\{0, \mathrm{a}_{0}: 0, \mathrm{a}_{0}: 1, \mathrm{a}_{1}: 0, \mathrm{a}_{1}: 1,1\right\}$ with $0 \otimes x=x$ and $\mathrm{a}_{i}: 0 \oslash \mathrm{a}_{i}: 1=1$. This is the algebra for Alice's measurements. Similarly, let $E_{\mathrm{B}}$ be the algebra for Bob's measurements. A distribution on the joint measurements of Alice and Bob is a bimorphism $E_{\mathrm{A}}, E_{\mathrm{B}} \rightarrow[0,1]$. We now give an elementary 
description of these bimorphisms. Each bimorphism $t: E_{\mathrm{A}}, E_{\mathrm{B}} \rightarrow[0,1]$ restricts to a function

$$
\tau:\left\{\mathrm{a}_{0}: 0, \mathrm{a}_{0}: 1, \mathrm{a}_{1}: 0, \mathrm{a}_{1}: 1\right\} \times\left\{\mathrm{b}_{0}: 0, \mathrm{~b}_{0}: 1, \mathrm{~b}_{1}: 0, \mathrm{~b}_{1}: 1\right\} \rightarrow[0,1]
$$

which we call a probability table, and we characterize these:

Proposition 10 A table $\tau:\left\{\mathrm{a}_{0}: 0, \mathrm{a}_{0}: 1, \mathrm{a}_{1}: 0, \mathrm{a}_{1}: 1\right\} \times\left\{\mathrm{b}_{0}: 0, \mathrm{~b}_{0}: 1, \mathrm{~b}_{1}: 0, \mathrm{~b}_{1}: 1\right\} \rightarrow[0,1]$ arises as the restriction of a bimorphism $E_{\mathrm{A}}, E_{\mathrm{B}} \rightarrow[0,1]$ if and only if

- it is a probability: $\sum_{o, o^{\prime} \in\{0,1\}} \tau\left(\mathrm{a}_{i}: o, \mathrm{~b}_{j}: o^{\prime}\right)=1$, for $i, j \in\{0,1\}$.

- it has marginalization, aka no signalling: for all $i, j \in\{0,1\}$,

$$
\begin{aligned}
\tau\left(\mathrm{a}_{i}: j, \mathrm{~b}_{0}: 0\right)+\tau\left(\mathrm{a}_{i}: j, \mathrm{~b}_{0}: 1\right) & =\tau\left(\mathrm{a}_{i}: j, \mathrm{~b}_{1}: 0\right)+\tau\left(\mathrm{a}_{i}: j, \mathrm{~b}_{1}: 1\right), \\
\tau\left(\mathrm{a}_{0}: 0, \mathrm{~b}_{i}: j\right)+\tau\left(\mathrm{a}_{0}: 1, \mathrm{~b}_{i}: j\right) & =\tau\left(\mathrm{a}_{1}: 0, \mathrm{~b}_{i}: j\right)+\tau\left(\mathrm{a}_{1}: 1, \mathrm{~b}_{i}: j\right) .
\end{aligned}
$$

The standard Bell table is as below, and by Proposition 10 it extends to a bimorphism $E_{\mathrm{A}}, E_{\mathrm{B}} \rightarrow[0,1]$. In this simple scenario we have two observers, each with two measurement settings, each with two outcomes, but it is straightforward to generalize to more elaborate Bell-like settings.

\begin{tabular}{l|cccc}
$t$ & $\mathrm{a}_{0}: 0$ & $\mathrm{a}_{0}: 1$ & $\mathrm{a}_{1}: 0$ & $\mathrm{a}_{1}: 1$ \\
\hline $\mathrm{b}_{0}: 0$ & $\frac{1}{2}$ & 0 & $\frac{3}{8}$ & $\frac{1}{8}$ \\
$\mathrm{~b}_{0}: 1$ & 0 & $\frac{1}{2}$ & $\frac{1}{8}$ & $\frac{3}{8}$ \\
$\mathrm{~b}_{1}: 0$ & $\frac{3}{8}$ & $\frac{1}{8}$ & $\frac{1}{8}$ & $\frac{3}{8}$ \\
$\mathrm{~b}_{1}: 1$ & $\frac{1}{8}$ & $\frac{3}{8}$ & $\frac{3}{8}$ & $\frac{1}{8}$
\end{tabular}

\subsection{Realization and Bell's paradox}

Quantum realization. A table has a 'quantum realization' if there is a way to obtain it by performing quantum experiments. Recall that a quantum system is modelled by a Hilbert space $\mathcal{H}$, and a yes-no question such as "is the outcome of measuring $\mathrm{a}_{0}$ equal to 1 " is given by a projection on this Hilbert space. The projections form an effect algebra $\operatorname{Proj}(\mathcal{H})$.

Definition 11 A quantum realization for a distribution on joint measurements $t: E, E^{\prime} \rightarrow[0,1]$ is given by finite dimensional Hilbert spaces $\mathcal{H}, \mathcal{H}^{\prime}$, two PPCM maps $r: E \rightarrow \operatorname{Proj}(\mathcal{H})$ and $r^{\prime}: E^{\prime} \rightarrow \operatorname{Proj}\left(\mathcal{H}^{\prime}\right)$, and a bimorphism $p:$ $\operatorname{Proj}(\mathcal{H}), \operatorname{Proj}\left(\mathcal{H}^{\prime}\right) \rightarrow[0,1]$, such that for all $e \in E$ and $e^{\prime} \in E^{\prime}$ we have $p\left(r(e), r^{\prime}\left(e^{\prime}\right)\right)=t\left(e, e^{\prime}\right)$.

The Bell table (4) has a quantum realization, with $\mathcal{H}=\mathcal{H}^{\prime}=\mathbb{C}^{2}$.

Classical realization. Classically, every time Alice and Bob perform a measurement, nature determines an assignment of outcomes for all measurements, which determines the outcomes for Alice and Bob. In such a deterministic theory we can calculate a probability for things like $\mathrm{a}_{0}: 0 \wedge \mathrm{a}_{1}: 1 \wedge \mathrm{b}_{0}: 1 \wedge \mathrm{b}_{1}: 1$, in which case if Alice chose $\mathrm{a}_{0}$ and Bob chose $\mathrm{b}_{1}$, they would get the outcome 0 and 1 , respectively. It can be shown (e.g., see [1]), that this is not the case for the standard Bell table. 
Definition $12 A$ classical realization for a distribution $t: E, E^{\prime} \rightarrow[0,1]$ is given by two Boolean algebras $B, B^{\prime}$, two effect algebra morphisms $r: E \rightarrow B$, $r^{\prime}: E^{\prime} \rightarrow B^{\prime}$ and a bimorphism $p: B, B^{\prime} \rightarrow[0,1]$ such that for all $e \in E$ and $e^{\prime} \in E^{\prime}$ we have $p\left(r(e), r^{\prime}\left(e^{\prime}\right)\right)=t\left(e, e^{\prime}\right)$.

Consider the Boolean algebra, $B_{\mathrm{A}}$, with atoms $\left\{\mathrm{a}_{1}: i \wedge \mathrm{a}_{2}: j \mid i, j \in\{0,1\}\right\}$. Note that $B_{\mathrm{A}}$ is a free completion of the effect algebra $E_{\mathrm{A}}$ to a Boolean algebra, in that, under identification of $\left(\mathrm{a}_{1}: 0 \wedge \mathrm{a}_{2}: 0\right) \vee\left(\mathrm{a}_{1}: 0 \wedge \mathrm{a}_{2}: 1\right)$ with $\mathrm{a}_{1}: 0$, we have $E_{\mathrm{A}} \subseteq B_{\mathrm{A}}$ and every morphism $E_{\mathrm{A}} \rightarrow B$, with $B$ a Boolean algebra, must factor through $B_{\mathrm{A}}$. Similarly, we have the algebra $B_{\mathrm{B}}$ for Bob.

Proposition 13 The canonical maps $r_{\mathrm{A}}: E_{\mathrm{A}} \rightarrow B_{\mathrm{A}}$ and $r_{\mathrm{B}}: E_{\mathrm{B}} \rightarrow B_{\mathrm{B}}$ cannot be completed to a classical realization of Table 4. Therefore, Table 4 has no classical realization.

\subsection{Tensor products}

Definition 14 The tensor product of two PPCMs E, $E^{\prime}$ is given by a PPCM $E \otimes E^{\prime}$ and a bimorphism $i: E, E^{\prime} \rightarrow E \otimes E^{\prime}$, such that for every bimorphism $f: E, E^{\prime} \rightarrow F$ there is a unique morphism $g: E \otimes E^{\prime} \rightarrow F$ such that $f=g \circ i$.

This gives a bijective correspondence between morphisms $E \otimes E^{\prime} \rightarrow F$ and bimorphisms $E, E^{\prime} \rightarrow F$. In fact, all tensor products of effect algebras exist (see e.g. [11]; but they can be trivial [8]). We return to the example of Alice and Bob.

Proposition 15 - The tensor product of Boolean algebras, $B_{\mathrm{A}} \otimes B_{\mathrm{B}}$, is the free Boolean algebra on the four elements $\left\{\mathrm{a}_{1}, \mathrm{a}_{2}, \mathrm{~b}_{1}, \mathrm{~b}_{2}\right\}$, where we identify, for example, $\mathrm{a}_{1}: 1$ with $\mathrm{a}_{1}$ and $\mathrm{a}_{1}: 0$ with $\neg \mathrm{a}_{1}$.

- The tensor product of effect algebras $E_{\mathrm{A}} \otimes E_{\mathrm{B}}$ is the effect algebra generated by the 16 elements $\mathrm{a}_{i}: 0 \wedge \mathrm{b}_{j}: 0, \mathrm{a}_{i}: 0 \wedge \mathrm{b}_{j}: 1, \mathrm{a}_{i}: 1 \wedge \mathrm{b}_{j}: 0, \mathrm{a}_{i}: 1 \wedge \mathrm{b}_{j}: 1$, for $i, j \in\{0,1\}$, such that each 4-tuple $\left(\mathrm{a}_{i}: 0 \wedge \mathrm{b}_{j}: 0, \mathrm{a}_{i}: 0 \wedge \mathrm{b}_{j}: 1, \mathrm{a}_{i}: 1 \wedge \mathrm{b}_{j}: 0, \mathrm{a}_{i}: 1 \wedge \mathrm{b}_{j}: 1\right)$ with $i, j \in\{0,1\}$ is a 4 -test. (For elements in such a 4-test we have that the effect algebra sum $\otimes$ is the Boolean join, $\vee$. Elements in different 4 -tests are not perpendicular.)

The statement of Bell's paradox can now be written in terms of homomorphisms, rather than bimorphisms:

Corollary 16 Table 4, $t: E_{\mathrm{A}} \otimes E_{\mathrm{B}} \rightarrow[0,1]$, does not factor through the embed$\operatorname{ding} E_{\mathrm{A}} \otimes E_{\mathrm{B}} \rightarrow B_{\mathrm{A}} \otimes B_{\mathrm{B}}$.

\subsection{Sheaf theoretic characterization}

Since the test functor $T: \mathbf{E A} \rightarrow \mathbf{S e t}^{\mathbb{N}}$ is full and faithful from effect algebras (Cor. 8), we can apply it to our effect algebra formulation of the Bell scenario, and arrive at a similar statement in terms of presheaves. Recall that $T([0,1])=D$, the distributions functor, and so the Bell table yields a natural transformation 
$T\left(E_{\mathrm{A}} \otimes E_{\mathrm{B}}\right) \rightarrow D$. Recall that $T\left(B_{\mathrm{A}} \otimes B_{\mathrm{B}}\right)=\mathbb{N}(16,-)$, the representable functor, and so the non-existence of a classical realization (Cor. 16) amounts to the nonexistence of a natural transformation as in the following diagram:

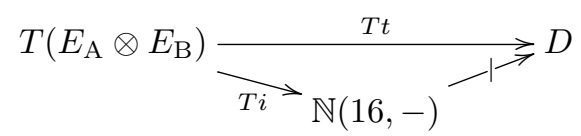

We can thus phrase Bell's paradox in the language of Grothendieck's sheaf theory. Since $i:\left(E_{\mathrm{A}} \otimes E_{\mathrm{B}}\right) \rightarrow\left(B_{\mathrm{A}} \otimes B_{\mathrm{B}}\right)$ is a subalgebra and $T$ preserves monos, $T\left(E_{\mathrm{A}} \otimes E_{\mathrm{B}}\right)$ is a subpresheaf of $\mathbb{N}(16,-)$, aka a 'sieve' on 16. A map $T\left(E_{\mathrm{A}} \otimes E_{\mathrm{B}}\right) \rightarrow D$ out of a sieve is called a 'compatible family', and a map $\mathbb{N}(16,-) \rightarrow D$ amounts to a distribution in $D(16)$ (by the Yoneda lemma). Bell's paradox now states: "the compatible family $T(t)$ has no amalgamation".

\subsection{Relationship with the work of Abramsky and Brandenburger}

Abramsky and Brandenburger [1] also phrase Bell's paradox in terms of a compatible family with no amalgamations. We now relate our statement with theirs.

Transferring the paradox to other categories. We can use adjunctions to transfer statements of non-factorization (such as Corollary 16) between different categories. Let $\mathcal{C}$ be a category and let $R: \mathbf{E A} \rightarrow \mathcal{C}$ be a functor with a left adjoint $L: \mathcal{C} \rightarrow$ EA. Let $j: X \rightarrow Y$ be a morphism in $\mathcal{C}$, and let $f: L(X) \rightarrow A$ be a morphism in EA. Then $f$ factors through $L(j)$ if and only if $f^{\sharp}: X \rightarrow R(A)$ factors through $j$, where $f^{\sharp}$ is the transpose of $f$.
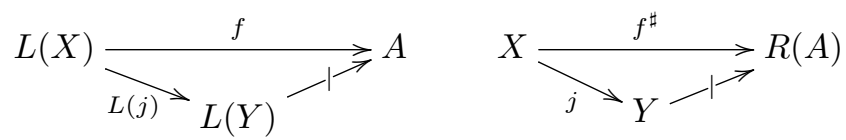

We use this technique to derive several equivalent statements of Bell's paradox. To start, the equivalence of the non factoring of the triangles (5) and (2) is immediate from the adjunction between the test functor and its left adjoint.

No global section. Recall that if $X$ is an object of a category $\mathcal{C}$ then the objects of the slice category $\mathcal{C} / X$ are pairs $(C, f)$ where $f: C \rightarrow X$. Morphisms are commuting triangles. The slice category $\mathcal{C} / X$ always has a terminal object, $\left(X, \operatorname{id}_{X}\right)$. The projection map $\Sigma_{X}: \mathcal{C} / X \rightarrow \mathcal{C}$, with $\Sigma_{X}(C, f)=C$, has a right adjoint $\Delta_{X}: \mathcal{C} \rightarrow \mathcal{C} / X$ with $\Delta_{X}(C)=\left(C \times X, \pi_{2}\right)$. First, notice that, using the adjunction $\Sigma_{\mathbb{N}(16,-)} \dashv \Delta_{\mathbb{N}(16,-)}$ we can rewrite diagram (5) in the slice category $\left(\mathbf{S e t}^{\mathbb{N}}\right) / \mathbb{N}(16,-)$ as:

$$
\left(T\left(E_{\mathrm{A}} \otimes E_{\mathrm{B}}\right), T i\right) \longrightarrow \frac{\langle T t, T i\rangle}{\longrightarrow} \longrightarrow(\mathbb{N}(16,-), \mathrm{id}) \longrightarrow\left(\longrightarrow \mathbb{N}(16,-), \pi_{2}\right)
$$

Since $(\mathbb{N}(16,-)$, id $)$ is terminal, we can phrase Bell's paradox as "the local section $\langle T t, T i\rangle:\left(T\left(E_{\mathrm{A}} \otimes E_{\mathrm{B}}\right), T i\right) \rightarrow\left(D \times \mathbb{N}(16,-), \pi_{2}\right)$ has no global section". 
Measurement covers. The analysis of Abramsky and Brandenburger is based on a 'measurement cover', which corresponds to our effect algebra $E_{\mathrm{A}} \otimes E_{\mathrm{B}}$.

Fix a finite set $X$ of measurements. In our Bell example, $X=\left\{\mathrm{a}_{0}, \mathrm{a}_{1}, \mathrm{~b}_{0}, \mathrm{~b}_{1}\right\}$. Also fix a finite set of $O$ of outcomes. In our example, $O=\{0,1\}$, so $O^{X}=16$. Abramsky and Brandenburger work in the category of presheaves $\mathcal{P}(X)^{\text {op }} \rightarrow$ Set on the powerset $\mathcal{P}(X)$ (ordered by subset inclusion). They explain Bell-type paradoxes as statements that a certain compatible family for the presheaf $D\left(O^{(-)}\right): \mathcal{P}(X)^{\text {op }} \rightarrow$ Set does not have a global section:

$$
\mathcal{M} \underset{+1 \rightarrow}{\longrightarrow} D\left(O^{(-)}\right)
$$

Here 1 is the terminal presheaf. The 'measurement cover' $\mathcal{M} \subseteq 1$ is defined by $\mathcal{M}(S)=\emptyset$ if $\left\{\mathrm{a}_{0}, \mathrm{a}_{1}\right\} \subseteq S$ or $\left\{\mathrm{b}_{0}, \mathrm{~b}_{1}\right\} \subseteq S$, and $\mathcal{M}(S)=\{*\}$ otherwise. In general, $\mathcal{M}(S)$ is inhabited, i.e., non-empty, if the measurement context $S$ is allowed in the Bell situation.

We now relate this diagram (7) with our diagram (2) by using an adjunction between $\mathbf{E A}$ and $\operatorname{Set}^{\mathcal{P}(X)^{\mathrm{op}}}$. We construct this adjunction as the following composite:

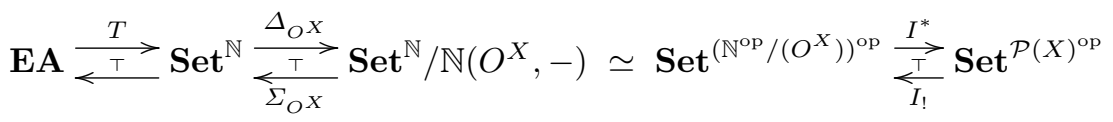

The first two adjunctions in this composite have already been discussed. The categorical equivalence $\mathbf{S e t}^{\mathbb{N}} / \mathbb{N}(16,-) \simeq \operatorname{Set}^{\left(\mathbb{N}^{\mathrm{op}} / 16\right)^{\mathrm{op}}}$ is an instance of a general fact about slices by representable presheaves (e.g. [13, Prop. A.1.1.7, Lem. C2.2.17]): in general, Set $^{\mathbb{D}^{\mathrm{op}}} / \mathbb{D}(-, d) \simeq$ Set $^{(\mathbb{D} / d)^{\mathrm{op}}}$.

It remains to explain $I_{!} \dashv I^{*}$. The functor $I^{*}: \mathbf{S e t}^{\left(\mathbb{N}^{\mathrm{op}} / 16\right)^{\mathrm{op}}} \rightarrow \mathbf{S e t}^{\mathcal{P}(X)^{\mathrm{op}}}$ is induced by precomposing with the functor $I: \mathcal{P}(X) \rightarrow \mathbb{N}^{\text {op }} / O^{X}$ that takes a subset $U \subseteq X$ to the pair $\left(O^{U}, O^{i_{U}}: O^{X} \rightarrow O^{U}\right)$ where $i_{U}: U \rightarrow X$ is the set inclusion function. It has a left adjoint, $I_{!}$, for general reasons (e.g. [13, Prop. A.4.1.4]).

Corollary 17 The right adjoint in (8) takes the effect algebra [0,1] to the presheaf $D\left(O^{(-)}\right)$: $\operatorname{Set}^{\mathcal{P}(X)^{\mathrm{op}}}$. The left adjoint in (8) takes the measurement cover $\mathcal{M} \subseteq 1$ to the effect algebra $E_{\mathrm{A}} \otimes E_{\mathrm{B}} \subseteq B_{\mathrm{A}} \otimes B_{\mathrm{B}}$.

Thus the adjunction (8) relates the effect algebra formulation of Bell's paradox (2), with the formulation of Abramsky and Brandenburger (7).

\subsection{Hardy paradoxes}

We now briefly consider a different kind of distribution. Not one where the entries are probabilities in the interval $[0,1]$, but where they are possibilities, i.e., either 1 for "this outcome is possible" or 0 for "this outcome is not possible". The pointed monoid $(\{0,1\}, \vee, 0,1)$ is built from these two possibilities. For an effect algebra 
$A$, a possibility distribution is a morphism $A \rightarrow(\{0,1\}, \vee, 0,1)$, and a possibility distribution on joint measurements is a bimorphism $A, B \rightarrow(\{0,1\}, \vee, 0,1)$.

The PPCM morphism $s:([0,1],+, 0,1) \rightarrow(\{0,1\}, \vee, 0,1)$ given by $s(0)=0$, $s(x)=1$ for $(x \neq 0)$ takes a probability distribution to its support, and by composing this with a probability distribution we get a possibility distribution.

The Hardy paradox concerns possibility, rather than probability. We can analyze it using PPCMs in a similar way to the way we analyzed the Bell paradox in Section 4.2. We can also relate our analysis with the analysis of Abramsky and Brandenburger [1], by embedding it in the presheaf category $\mathbf{S e t}^{\mathbb{N}}$. Here the situation is slightly more subtle: we cannot use Corollary 8 since $(\{0,1\}, \vee, 0,1)$ is not an effect algebra, but we can still use Theorem 7 , since it only appears on the right-hand-side of arrows.

\subsection{Kochen-Specker systems}

A Kochen-Specker system is represented by a sub-effect algebra $E$ of $\operatorname{Proj}(\mathcal{H})$

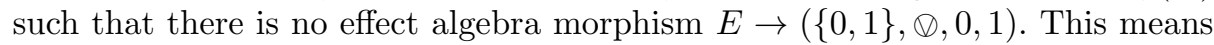
we cannot assign a value 0 or 1 to every element of $E$ in such a way that whenever $p_{1}, \ldots p_{n} \in E$ with $p_{1}+\ldots p_{n}=1$, exactly one of the $p_{i}$ is assigned 1 and this assignment does not depend on the other $p_{j}, j \neq i$. (NB here we use partial join $\oslash$, with $1 \otimes 1$ undefined, whereas we used the total join $\vee$ in $\S 4.6$.)

We now view this in the presheaf category Set $^{\mathbb{N}}$. Since there is no morphism $\operatorname{Proj}(\mathcal{H}) \rightarrow(\{0,1\}, \oplus, 0,1)$, there is also no natural transformation $T(\operatorname{Proj}(\mathcal{H})) \rightarrow$ $T(\{0,1\}, \otimes, 0,1)$, by Corollary 8 . We now explore this more explicitly.

The bounded operators on $\mathcal{H}$ form a $\mathrm{C}^{*}$-algebra, $B(\mathcal{H})$. An $n$-test in the effect algebra $\operatorname{Proj}(\mathcal{H})$ can be identified with a unital ${ }^{*}$-homomorphism $\mathbb{C}^{n} \rightarrow B(\mathcal{H})$ from the commutative $\mathrm{C}^{*}$-algebra $\mathbb{C}^{n}$, by looking at the images of the characteristic functions on single points. So $T(\operatorname{Proj}(\mathcal{H})) \cong \mathbf{C}^{*}\left(\mathbb{C}^{-}, B(\mathcal{H})\right)$. On the other hand, $T(\{0,1\}, \oslash, 0,1)(N)=N$.

There is another way to view this, via a restricted Gelfand duality. Let $\mathbf{C C}_{\mathrm{f}}^{*}$ be the category of finite dimensional commutative $\mathrm{C}^{*}$-algebras. The functor $\mathbb{C}^{-}: \mathbb{N}^{\text {op }} \rightarrow \mathbf{C C}_{\mathrm{f}}^{*}$ is an equivalence of categories. Under this equivalence we have presheaves $T(\operatorname{Proj}(\mathcal{H})), T(\{0,1\}, \otimes, 0,1) \in \mathbf{S e t}^{\mathbf{C C}_{\mathrm{f}}^{* \text { op }}}$ with

$$
T(\operatorname{Proj}(\mathcal{H}))(A)=\mathbf{C}^{*}(A, B(\mathcal{H})) \quad T(\{0,1\}, \otimes, 0,1)(A)=\operatorname{Spec}(A)
$$

where $\operatorname{Spec}(A)$ is the Gelfand spectrum of $A$. Thus the Kochen-Specker paradox says that there is no natural transformation $\mathbf{C}^{*}(-, B(\mathcal{H})) \rightarrow$ Spec in $\mathbf{S e t}^{\mathbf{C C}_{\mathrm{f}}^{* \text { op }}}$.

We can use adjunctions to transport this statement to other categories. If a functor $R:$ Set $^{\mathbf{C C}_{\mathrm{f}}^{* \text { op }}} \rightarrow \mathcal{C}$ has a left adjoint $L: \mathcal{C} \rightarrow$ Set $^{\text {CC }_{\mathrm{f}}^{* \text { op }}}$ and $L(X)=$ $\mathbf{C}^{*}(-, B(\mathcal{H}))$ then the paradox says there is no morphism $X \rightarrow R$ (Spec) in $\mathcal{C}$.

In particular, we transport the paradox to the setting of Hamilton et al. [10], who were concerned with presheaves on the poset $C(B(\mathcal{H}))$ of commutative subalgebras of $B(\mathcal{H})$. We do this using the following composite adjunction:

$$
\mathbf{S e t} \underset{\Sigma_{\mathbf{C}^{*}(-, B(\mathcal{H}))}}{\stackrel{\mathbf{C C}_{\mathrm{f}}^{*} \mathbf{C}^{*}(-, B(\mathcal{H}))}{\underset{T}{\longrightarrow}}} \mathbf{S e t}^{\mathbf{C C}_{\mathrm{f}}^{* \mathrm{op}}} / \mathbf{C}^{*}(-, B(\mathcal{H})) \simeq \mathbf{S e t}^{\left(\mathbf{C C}_{\mathrm{f}}^{*} \downarrow B(\mathcal{H})\right)^{\mathrm{op}}} \underset{J_{!}}{\stackrel{J^{*}}{T}} \mathbf{S e t}^{C(B(\mathcal{H}))^{\mathrm{op}}}
$$


The first adjunction between slice categories is as in Section 4.5. The middle equivalence is standard (e.g. [13, Prop. A.1.1.7]); here $\left(\mathbf{C C}_{\mathrm{f}}^{*} \downarrow B(\mathcal{H})\right)$ is the category whose objects are pairs $(A, f: A \rightarrow B(\mathcal{H}))$ where $A$ is a finite-dimensional commutative $\mathrm{C}^{*}$-algebra and $f$ is a ${ }^{*}$-homomorphism. The adjunction $J_{!} \dashv J^{*}$ is induced by the evident embedding $J: C(B(\mathcal{H})) \rightarrow\left(\mathbf{C C}_{\mathrm{f}}^{*} \downarrow B(\mathcal{H})\right)$.

The left adjoint of this composite takes the terminal presheaf on $C(B(\mathcal{H}))$ to the presheaf $\mathbf{C}^{*}(-, B(\mathcal{H}))$ on $\mathbf{C C}_{\mathrm{f}}^{*}$. The right adjoint takes the spectral presheaf on $\mathbf{C C}_{\mathrm{f}}^{*}$ to the spectral presheaf on $C(B(\mathcal{H}))$. Thus our statement of the paradox is equivalent to the statement of [10]: the spectral presheaf has no global section.

Summary. We have exhibited a crucial adjunction between two general approaches to finite probability theory: effect algebras and presheaves (Corollary 8). We have used this to analyze paradoxes of non-locality and contextuality (Section 4). There are simple algebraic statements of these paradoxes in terms of partial commutative monoids, but these transport across the adjunction to statements about presheaves on $\mathbb{N}$. By taking slice categories of the presheaf category, we recover earlier analyses of the paradoxes (e.g. Corollary 17).

Acknowledgment. We thank Robin Adams, Tobias Fritz, Bart Jacobs, Frank Roumen, the Royal Society and the ERC.

\section{References}

1. Abramsky, S., Brandenburger, A.: The sheaf-theoretic structure of non-locality and contextuality. New J. Phys 13 (2011)

2. Adámek, J., Rosický, J.: Locally Presentable and Accessible Categories. CUP

3. Brotherston, J., Calcagno, C.: Classical BI: Its semantics and proof theory. Log. Meth. Comput. Sci. 6(3) (2010)

4. Calcagno, C., O'Hearn, P.W., Yang:, H.: Local action and abstract separation logic. In: Proc. LICS 2007. pp. 366-378 (2007)

5. Dvurečenskij, A., Pulmannová, S.: New trends in quantum structures. Kluwer

6. Engesser, K., Gabbay, D.M., Lehmann, D. (eds.): Handbook of Quantum Logic and Quantum Structures: Quantum Structures. Elsevier (2007)

7. Fiore, M.P., Plotkin, G.D., Turi, D.: Abstract syntax and variable binding. In: Proc. LICS 1999 (1999)

8. Foulis, D.J., Bennett, M.K.: Tensor products of orthoalgebras. Order 10, 271-282

9. Foulis, D.J., Bennett, M.: Effect algebras and unsharp quantum logics. Found. Physics 24(10), 1331-1352 (1994)

10. Hamilton, J., Isham, C.J., Butterfield, J.: Topos perspective on the Kochen-Specker theorem: III. Int. J. Theoret. Phys. pp. 1413-1436 (2000)

11. Jacobs, B., Mandemaker, J.: Coreflections in algebraic quantum logic. Foundations of physics 42(7), 932-958 (2012)

12. Jacobs, B.: Probabilities, distribution monads, and convex categories. Theor. Comput. Sci. 2(28) (2011)

13. Johnstone, P.T.: Sketches of an elephant: a topos theory compendium. OUP (2002)

14. Kelly, G.M.: Basic concepts of enriched category theory. CUP (1980)

15. Mac Lane, S., Moerdijk, I.: Sheaves in geometry and logic. Springer-Verlag (1992)

16. Staton, S.: Instances of computational effects. In: Proc. LICS 2013 (2013) 